\title{
Correlation of Peritumoral Edema and Microvessel Density with Tissue Expression of VEGF, Semaphorins 3A and 3C in Patients with Meningioma
}

\author{
Mehdi Salimi-Sotoodeh ${ }^{1}$, Arash Saffarian ${ }^{1}$, Mousa Taghipour ${ }^{1}$, Amir-Reza \\ Dehghanian $^{2}$, Nooshafarin Chenari ${ }^{3}$,Abbas Ghaderi' ${ }^{3,4}$, Mahboobeh Razmkhah ${ }^{3}$
}

'Department of Neurosurgery, School of Medicine, Shiraz University of Medical Sciences, Shiraz, Iran. ${ }^{2}$ Department of Pathology, School of Medicine, Shiraz University of Medical Sciences, Shiraz, Iran. ${ }^{3}$ Shiraz Institute for Cancer Research, School of Medicine, Shiraz University of Medical Sciences, Shiraz, Iran. ${ }^{4}$ Department of Immunology, School of Medicine, Shiraz University of Medical Sciences, Shiraz, Iran.

\begin{abstract}
Background: Several angiogenic factors correlate with angiogenesis in meningioma while their exact role is yet to be identified. Semaphorins are described with a variety of physiological functions including angiogenesis and migration of neural crest cells. Objective: We aimed to determine the correlation of semaphorin 3A, 3C (Sema $3 \mathrm{~A}$ and $3 \mathrm{C}$ ) and vascular endothelial growth factor (VEGF) expression with microvessel density (MVD) and peritumoral brain edema (PTBE) in meningioma. Methods: In this study 21 patients with grade I meningioma were included. PTBE was measured on axial and coronal brain MRI. Tissue expression of semaphorin 3A, 3C and VEGF were determined using quantitative real-time PCR (qRT-PCR). Result: We found that mean vascular density and tumor edema index were negatively associated with tissue expression of semaphorin $3 \mathrm{~A}$ and $3 \mathrm{C}$, respectively $(\mathrm{p}=0.029$ and $\mathrm{p}=0.048)$. VEGF did not show statistically significant correlation with tumor characteristics studied ( $>0.05$ ). We also found that the mean vascular density of the menigiomas was positively associated with intra operative blood loss $(\mathrm{r}=0.503, \mathrm{p}=0.010)$. Conclusion: Our data indicates an inverse correlation of Sema3A and Sema3C with vascular density and peritumoral edema, respectively, while no correlation could be shown for VEGF. Thus, Sema3A and 3C may be identified as appropriate inhibitors of pathological angiogenesis in human meningioma. However, confirmation of this finding in a larger dataset is warranted.
\end{abstract}

Keywords: Meningioma- Semaphorins- Vascular endothelial growth factor- Peritumoral brain edema- Microvessel density

Asian Pac J Cancer Biol, 3 (4), 93-98

\section{Introduction}

Meningioma is an extra-axial tumor without symptoms unless it makes pressure effect on functional areas in CNS. Some meningiomas are clinically offensive and can contribute to considerable complications and even death [1-2]. Angiogenesis has been reportedly correlated with tumor recurrence and shorter overall survival in meningioma patients. As a consequence, neoangiogenesis may have a potential implication in offering novel treatment modalities to decrease the growth of meningioma or recurrence risk [3-4]. Angiogenesis is related to the equivalence between
Submission Date: 10/05/2018 Acceptance Date: 12/06/2018

angiogenic and anti-angiogenic regulators. It is widely accepted that vascular endothelial growth factor (VEGF) plays a radical role in the stimulation of angiogenesis by enhancing the migration, proliferation and tube formation of endothelial cells [5]. Upregulation of VEGF plays a prominent role as a pro-angiogenic factor for edema formation in meningioma. Indeed, preclinical data indicated that there may be correlation between VEGF expression and higher grades of meningioma [6-8]. The pro-angiogenic activity of VEGF may be antagonized by the anti-angiogenic factor semaphorin3 A (Sema3A). Sema3A is an axon guidance protein that belongs to the class 3 semaphorins (Semas) and acts

Corresponding Author:

Mahboobeh Razmkhah

Shiraz Institute for Cancer Research, School of Medicine, Shiraz University of Medical Sciences, Shiraz, Iran.

Email: razmkhahm@sums.ac.ir 
through the binding to neuropilin-1 (NRP-1), a protein primarily identified on neuronal cells and also expressed on the endothelial cells as a trans-membrane receptor. The binding of Sema3A to NRP-1 contributes to apoptosis and suppression of endothelial cells proliferation [9]. Sema3A expression was recently shown in the neoplastic cells of human meningiomas and it has been suggested to behave as an anti-angiogenic factor in this neoplasia [10]. Therefore, neoangiogenesis in meningioma is regulated by the balance in the concentrations of both VEGF and Sema3A in the microenvironment of the tumor, rather than by VEGF alone [9]. In particular, different semaphorins can either raise or prevent tumor progression processes such as tumor angiogenesis, tumor metastasis and tumor cell survival [11]. Most of the class 3 semaphorins (semaphorins 3A, 3B, 3D, 3F and 3G) were demonstrated to inhibit cell migration and characterized with antitumor properties. However, semaphorin 3C (Sema3C) enhances tumor cell migration and is highly expressed in metastatic tumor cells [11]. Furthermore, Sema3C promotes endothelial cell proliferation, migration, and tube formation in vitro [12] but its concrete role in vivo is still debated.

Herein we sought to determine the correlation between Sema3A, Sema3C and VEGF mRNA expression and the microvessel density of the tumor and the grade of peritumoral brain edema (PTBE) in a series of human meningiomas.

\section{Materials and Methods}

\section{Demographic characteristics of patients}

Brain tumor tissues were obtained during a 1-year period from 2014 to 2015 from a total number of twenty one patients whom meningioma diagnosis was confirmed by preoperative imaging study and scheduled for tumor resection surgery in Shahid Chamran and Namazi Hospitals, Shiraz University of Medical Sciences, Shiraz, Iran. Patients with recurrent tumors, history of chemotherapy and radiotherapy and comorbid medical conditions (diabetes, rheumatoid arteritis, inflammatory diseases and addiction) were excluded from our study. Medical charts of the patients included were reviewed for demographic information including age and gender, tumor characteristics including duration of symptoms, neurologic examination, radiologic findings and intraoperative findings. Ethics committee of Shiraz University of Medical Sciences approved the study protocol (ethics code: 5598) and all participants provided their informed consent to take part in this study.

\section{RNA extraction and cDNA synthesis}

Tissue samples were immediately transferred into Stem Cell Laboratory of Shiraz Institute for Cancer Research, Shiraz University of Medical Sciences. Tissues were cut into small pieces and total RNA was extracted using appropriate Tissue RNA extraction kit (Invitrogen, Germany). cDNA was synthesized from the extracted RNAs using the cDNA synthesis kit according to the manufacturer's instructions (Fermentas, Lithuania).
Quantitative real time PCR ( $q R T-P C R)$

Human Sema3A and $3 C$ and VEGF gene transcripts were evaluated by an ABI thermal cycler (ABI, USA) for qRT-PCR. Each PCR reaction was carried out in a final volume of $20 \mu \mathrm{l}$ containing $2 \mu \mathrm{l} \mathrm{cDNA}, 10 \mu \mathrm{l}$ of $2 \mathrm{X}$ SYBR Green Master Mix (Fermentas, Canada), $0.3 \mu$ l of each 10 pmol forward and reverse primers and 7.4 $\mu$ LEPC treated water. Thermal cycling for all the genes was initiated with a denaturation step at $95^{\circ} \mathrm{C}$ for $10 \mathrm{~min}$, followed by 50 cycles with the subsequent program for each cycle: $95^{\circ} \mathrm{C}$ for $15 \mathrm{~s}, 57^{\circ} \mathrm{C}$ for $30 \mathrm{~s}$ and $60^{\circ} \mathrm{C}$ for 1 minute. All data were compared to those from $18 \mathrm{~S}$ rRNA housekeeping gene.

\section{Microvessel density count and peritumoral edema} estimation

Brain MRI of patients were assessed for acquisition of brain tumoral edema index (TEI); Meningioma tumoral volume was calculated in T1WI Gadolinium enhanced images measuring largest axial diameter as (A) perpendicular diameter in the same cut as (B) and largest coronal diameter as $(\mathrm{C})$ using sphere volume formula: volume $=4 / 3 \times \pi \times \mathrm{r}^{3}$ simplified as volume $\pi \times 1 / 6 \times \mathrm{d}^{3}$ in which $\mathrm{d} 3$ represented $\mathrm{A}^{*} \mathrm{~B}^{*} \mathrm{C}$. Peritumoral brain edema (PTBE) volume was calculated in similar way by measuring signal intensity diameter in axial and coronal T2WI images in which edema boarders could be clearly identified from normal tissues and this volume included the tumor. Tumor edema index (TEI) was obtained from dividing PTBE by tumor volume. The Edema index indicated the rate of PTBE, related to tumor volume, thus 1.0 was associated with no PTBE.

After acquisition of pathologic slides from tumor tissue blocks and confirming the diagnosis and evaluation of grading according to WHO 2016 grading [13], slides were evaluated for signs of neovascularization and angiogenesis. Briefly three most vascularized areas were detected and selected under low-power field $\left({ }^{\times} 40\right)$. Then microvessels were counted in each of these areas under a high-power field $\left({ }^{\times} 400\right)$.

\section{Statistical analysis}

All the data were analyzed using the statistical package for social sciences (SPSS Inc., Chicago, IL, USA) version 16.0. The correlation between Sema3A, 3C, VEGF, microvessel density (MVD) and peritumoral brain edema (PTBE) was assessed using Pearson's correlation. Linear regression model was performed to assess the correlation between tumor expression of these markers and PTBE and MVD. Independent t-test was used to compare the MVD, tumor expression of Sema3A, 3C and VEGF between those with and without PTBE. A two-sided p-value less than 0.05 was considered significant. The relative amounts of VEGF, Sema3A and 3C transcripts were determined using $2^{-\Delta \mathrm{Ct}}$ formula in meningioma patients. 


\section{Results}

\section{Demographic characteristics}

21 patients with grade I meningioma who underwent open surgery were included in this study. The mean age of meningioma patients were $55.2 \pm 13.5$ (ranging from 22 to 78 ). Demographic and clinical characteristics of patients studied are presented in Table 1.

The most common site of the meningioma in our population was found to be convexity in 7 (33.3\%) patients. The most common presenting symptoms were headache and blurred vision being recorded in $6(28.6 \%)$ patients followed by focal neurologic deficit in 4 (19.0\%) patients.

Tumor characteristics and tissue expression of angiogenic factors

Table 2 summarized the tumor characteristics of patients. The mean tumor volume was found to be $35793 \pm$ 37108.6 (range 1802.3 to 161000 ) $\mathrm{mm}^{3}$. The mean tumor edema index was reported to be $2.6 \pm 2.3$. The Mean vascular density was reported to be $20.6 \pm 10.8$ vessels per $\mathrm{mm}^{2}$ (10.3 \pm 5.3 vessels/HPF Olympus BX51, eyepiece magnification 10X20mm) (Figure 1).

The tissue expression of the angiogenic factors are also demonstrated in Table 2. The mean tissue expression of Sema3A, 3C and VEGF was reported to be $1.4099 \times 10^{-4}$, $2.2 \times 10^{-3}$, and $3.34 \times 10^{-6}$ (Table 2).

\section{Correlation analysis}

We performed bivariate correlation analysis in order to determine the linear correlation between the tumor characteristics and the tissue expression of the

Table 1. Demographic and Clinical Characteristics of 21 Patients with Meningioma

\begin{tabular}{lc}
\hline Variable & Value \\
\hline Age (years) & $55.2 \pm 13.5$ \\
Sex & \\
$\quad$ Men (\%) & $4(19.0 \%)$ \\
Women (\%) & $17(81.0 \%)$ \\
Location & \\
Convexity (\%) & $7(33.3 \%)$ \\
Sphenoid wing (\%) & $6(28.6 \%)$ \\
Parasagittal (\%) & $3(14.3 \%)$ \\
Tuberculumsella (\%) & $2(9.5 \%)$ \\
Tentorial (\%) & $2(9.5 \%)$ \\
Olfactory groove (\%) & $1(4.8 \%)$ \\
Presenting symptoms & \\
Headache (\%) & $6(28.6 \%)$ \\
Blurred vision (\%) & $6(28.6 \%)$ \\
Focal neurologic deficit (\%) & $4(19.0 \%)$ \\
Seizure (\%) & $3(14.3 \%)$ \\
Slurred speech (\%) & $1(4.8 \%)$ \\
Behavioral change (\%) & $1(4.8 \%)$ \\
\hline
\end{tabular}

Table 2. Tumor Characteristics and Tissue Expression of Angiogenic Factors in Meningioma Patients. HPF,high power field; Sema, semaphorin; VEGF, vascular endothelial growth factor.

\begin{tabular}{lc} 
& Grade $\mathrm{I}(\mathrm{n}=21)$ \\
\hline Tumor volume $\left(\mathrm{mm}^{3}\right)$ & $35793.3 \pm 37108$ \\
Tumor edema index & $2.56 \pm 2.33$ \\
Mean vascular density & $20.6 \pm 10.8$ \\
$\left(\right.$ vessel $\left./ \mathrm{mm}^{2}\right)$ & $(10.3 \pm 5.4$ vessels $/ \mathrm{HPF})$ \\
Angiogenesis index & $15676.3 \pm 62653$ \\
Intraoperative bleeding $(\mathrm{mL})$ & $1016.6 \pm 694.5$ \\
Sema3A & $1.4099^{\times} 10^{-4}$ \\
Sema3C & $2.2^{\times} 10^{-3}$ \\
VEGF & $3.34 \times 10^{-6}$ \\
\hline
\end{tabular}

angiogenic factors (Table 3). Both MVD and tumor edema index showed negative significant correlation with tissue expression of Sema3A $(r=-0.477, p=0.029)$ and Sema $3 \mathrm{C}(\mathrm{r}=-0.437, \mathrm{p}=0.048)$, respectively. No statistically significant correlation was observed between tissue expression of VEGF and tumor characteristics in meningioma ( $p>0.05)$. We also found that the mean vascular density of the menigiomas was positively associated with intraoperative blood loss $(\mathrm{r}=0.503$, $\mathrm{p}=0.010)$ (Figure 2).

\section{Peritumoral index}

We compared the demographic and tumor characteristics as well as tissue expression of Sema 3A and $3 \mathrm{C}$ and VEGF between those with peritumoral index higher than 2 and those with lower values. We found that there was no significant difference between those with peritumoral edema more than 2 and those with values less than 2 (Table 4).

\section{Discussion}

Meningiomas are among the most common intracranial tumors which are considered to be benign in nature and course [14]. However, their mass effect and PTBE is associated with focal neurologic deficit and associated

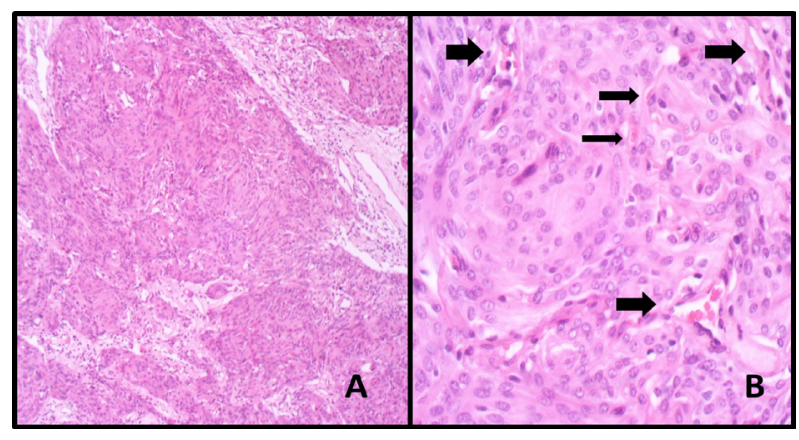

Figure 1. Histopathology Evaluation of Microvascular Density. A. Section Shows Sheets of Tumoral Cells in Syncytial Pattern and Vague Whirling (X100, Hematoxyllin and Eosin) B. Section Shows tumor with its microvasculature (Arrows) per one high power field. (X400, H\&E). 
Table 3. Correlation Analysis between Tissue Expression of the Semaphorins and VEGF and Tumor Characteristics in Meningioma. Sema, semaphorin; VEGF, vascular endothelial growth factor.

\begin{tabular}{lcc}
\hline & r-value & p-value \\
\hline Sema3A & & \\
Age (years) & -0.044 & 0.849 \\
Tumor volume $\left(\mathrm{mm}^{3}\right)$ & -0.035 & 0.880 \\
Tumor edema index & 0.104 & 0.653 \\
Mean vascular density & -0.477 & 0.029 \\
Intraoperative bleeding $(\mathrm{mL})$ & -0.233 & 0.308 \\
Sema3C & & \\
Age (years) & -0.045 & 0.847 \\
Tumor volume (mm $\left.{ }^{3}\right)$ & -0.110 & 0.634 \\
Tumor edema index & -0.437 & 0.048 \\
Mean vascular density & -0.089 & 0.703 \\
Intraoperative bleeding $(\mathrm{mL})$ & 0.023 & 0.922 \\
VEGF & & \\
Age (years) & 0.029 & 0.902 \\
Tumor volume (mm $\left.{ }^{3}\right)$ & -0.048 & 0.836 \\
Tumor edema index & -0.244 & 0.286 \\
Mean vascular density $\left(\mathrm{vessel} / \mathrm{mm}^{2}\right)$ & -0.151 & 0.513 \\
Intraoperative bleeding $(\mathrm{mL})$ & -0.269 & 0.239 \\
\hline
\end{tabular}

symptoms. These tumors are highly vascular and their operation is usually associated with significant intra operative bleeding. Several factors have been identified as the predictors of PTBE in human meningiomas including histological findings, age, gender and radiological definitions such as tumor size, vascularity, tumor location, and tumor shape [15-16]. Furthermore, it has been proposed that PTBE can be caused by increased permeability of the surrounding vasculature [16] along with a defect in the blood-brain-barrier [17]. Despite these mechanisms, the exact pathogenesis of PTBE is still a matter of debate. In the present study we purposed to determine the correlation between PTBE, microvessel density and tissue expression of VEGF as well as Sema3A and $3 \mathrm{C}$. This would enable us to better understand the pathophysiology of PTBE and tumoral angiogenesis.

Semaphorins were first described by Luo et al. as

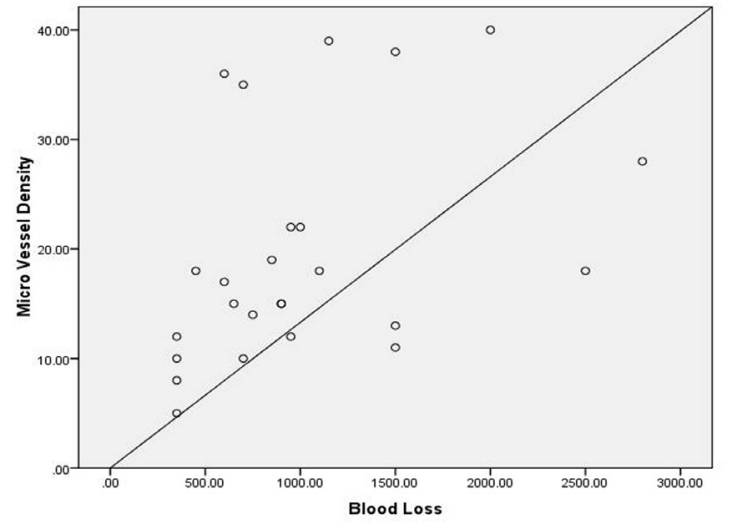

Figure 2. Scattered Plot for Linear Correlation between Microvessel Density and Intraoperative Blood Loss $(\mathrm{r}=0.503, \mathrm{p}=0.010)$.

prominent factors playing role in the development of nervous system during the embryogenic period [18]. Further, they were found to play several roles in cell migration, immune responses, tumor progression and angiogenesis. Sema3A has been reported to be an anti-angiogenic factor being involved in the regulation of the tumor growth and progression. This molecule was found to be an apoptosis inducer in endothelial cells [9] and recent in vivo experiments demonstrated that the addition of Sema3A is associated with inhibition of angiogenesis [19]. Barresi et al. showed that Sema3A expression in human meningioma is associated with low microvessel density and lower recurrence rate which clearly shows the antiangiogenic effects of Sema3A [9]. Our result of Sema3A is in consistent with these reports since we also found negative correlation between MVD and expression of Sema3A.

Prediction and management of PTBE is important in meningioma because most of meningiomas with PTBE are located in eloquent regions of the brain. As microvessel density is a predictor of PTBE, it is expected that angiogenic factors such as VEGF show positive correlation to PTBE [7-20]. In contrast, Sema3A was shown to be associated with low microvessel density of the tumor and in turn less PTBE [21]. Here we found that the meningioma microvessel density is positively correlated with intra operative bleeding. However, in

Table 4. Comparing the Tumor Characteristics and the Tissue Expression of the Sema3A and 3C and VEGF between those with Peritumoral Edema Index $\geq 2$ and $<2$. HPF, high power field; Sema, semaphorin; VEGF, vascular endothelial growth factor.

\begin{tabular}{lccc}
\hline & TEI $\geq 2$ & TEI $<2$ & p-value \\
\hline Age (years) & $54.5 \pm 14.4$ & $55.7 \pm 13.4$ & 0.84 \\
Tumor volume $\left(\mathrm{mm}^{3}\right)$ & $46709 \pm 51702$ & $27607 \pm 19724$ & 0.25 \\
Mean vascular density & $22.7 \pm 12.9$ & $19.1 \pm 9.5$ & 0.47 \\
$\left(\right.$ vessel $\left./ \mathrm{mm}^{2}\right)$ & $(11.3 \pm 6.3$ vessels/HPF $)$ & $(9.5 \pm 4.7$ vessels/HPF $)$ & \\
Intraoperative bleeding $(\mathrm{mL})$ & $1050 \pm 871$ & $992 \pm 569$ & 0.85 \\
Sema3A & $2.69 \times 10^{-4}$ & $4.5 \times 10^{-5}$ & 0.12 \\
Sema3C & $3.05 \times 10^{-3}$ & $1.71 \times 10^{-3}$ & 0.68 \\
VEGF & $9.4 \times 10^{-7}$ & $5.1 \times 10^{-6}$ & 0.35 \\
\hline
\end{tabular}


our study, neither Sema3A nor VEGF showed association with PTBE while Sema3C was inversely associated with PTBE. Although anti-angiogenic functions of Sema3 proteins such as Sema3A and Sema3F is determined [22], Sema3C is still a poorly understood molecule and there are conflicting reports in the literature regarding the function of this molecule. On one hand, some malignancies including glioma and gastric cancer display high expression of Sema3C; showing a potential role of this molecule in pathological conditions [23-24]. Moreover, Banu et al. showed that the Sema3C is associated with glomerular endothelial cell proliferation, migration and tube formation; however, the results were collected from in vitro studies [25]. On the other hand, Yang et al. marked Sema3C as an efficient mediator for suppressing pathological retinal angiogenesis [26]. Sema3C/Neuropilin-1/PlexinD1 signaling disturbs focal adhesions, cytoskeleton assembly and endothelial cell junctions, leading to the endothelial cell apoptosis and the reduced cell migration [27]. Interestingly, suppressing the formation of pre-retinal neovascular tufts through Sema3C may be more effective than using Sema3 receptor neutralizing antibodies [26-27]. Thus, Sema3C is suggested as a favorable inhibitor for pathological angiogenesis [26].

Collectively, the role of Sema3C may mostly depend on the type of the tumor as it is also reported for Sema3A which is shown to be an endogenous inhibitor of tumor progression [28] but not in glioblastoma multiform and pancreatic cancer [29]. Based on the present data it seems that Sema3C as well as other Sema3 members has anti-angiogenic functions in meningioma.

We did not find any correlation between tissue expression of Sema3 A and $3 \mathrm{C}$ with tumor size and intraoperative bleeding. One explanation could be the role of steroids. All the patients included in this study received intravenous steroid in order to decrease the intracranial pressure and PTBE. This might affect the correlation negatively. In addition, high doses of corticosteroids are associated with decrease vascular permeability which can interfere with VEGF effects and angiogenesis equation and in turn might alter the mediated proteins expression level [7].

We note some limitations to our study. First, the number of patients included in the current study was limited because our study was performed in a referral center in which the majority of meningioma cases were en plaque base skull meningioma with less peritumoral edema and minimal sample for tissue RNA extraction, which made a technical problem during study. Thus further research on a larger population is required for clinical conclusion. Second, we did not follow the patients to record the outcome, recurrence and occurrence of other neoplasms. As Li et al. indicated that expression of semaphorins is associated with recurrence rate, [30] we had better follow the patients to obtain data on this issue.

In conclusion, tissue expressions of Sema3 A and Sema3C in our meningioma patients were associated with decreased MVD and PTBE and the authors conclude that these two semaphorins may have inhibitory effects on angiogenesis in meningioma. Accordingly, Sema3A and 3C may be introduced as promising inhibitors for MVD and PTBE in meningioma patients in future. Nonetheless, further research is required to better elucidate the physiological and pathological functions of these molecules in different types of cancers.

\section{Acknowledgements}

The authors would like to thank all the participants in the study. This work was financially supported by a grant from Shiraz University of Medical Sciences and Shiraz Institute for Cancer Research [Grant No. 92-0101-5598 and ICR-100-504]. This research was done as a requirement for the neurosurgical specialty thesis performed by Dr. Mehdi Salimi-Setoodeh.

\section{Conflict of interest}

The authors confirm that there is no conflict of interest.

\section{References}

1. Rockhill J, Mrugala M, Chamberlain MC. Intracranial meningiomas: an overview of diagnosis and treatment. Neurosurg Focus 2007;23:E1.

2. Backer-Grøndahl T, Moen BH, Torp SH. The histopathological spectrum of human meningiomas. Int J Clin Exp Pathol 2012;5:231-242.

3. Barresi V. Angiogenesis in meningiomas. Brain Tumor Pathol 2011;28:99-106.

4. Kandemir NO, Narli ZI, Kalayci M, Ozdamar SO. A Rare Pattern of Angiogenesis in Meningiomas: Glomeruloid Microvascular Proliferation. Turk Neurosurg 2014;24:765769.

5. Nagane M. [Anti-angiogenic therapy for malignant glioma]. Gan to Kagaku Ryoho 2014;41:141-147.

6. Dharmalingam P, Kumar VR, Verma SK. Vascular endothelial growth factor expression and angiogenesis in various grades and subtypes of meningioma. Indian J Pathol Microbiol 2013;56:349.

7. Hou J, Kshettry VR, Selman WR, Bambakidis NC. Peritumoral brain edema in intracranial meningiomas: the emergence of vascular endothelial growth factor-directed therapy. Neurosurg Focus 2013;35:E2.

8. Nassehi D. Intracranial meningiomas, the VEGF-A pathway, and peritumoral brain oedema. Dan Med J 2013;60:B4626.

9. Barresi V, Tuccari G.Increased ratio of vascular endothelial growth factor to semaphorin $3 \mathrm{~A}$ is a negative prognostic factor in human meningiomas. Neuropathol 2010;30:537546.

10. Barresi V, Tuccari G. Evaluation of neo-angiogenesis in a case of chordoid meningioma. J Neurooncol 2009;95:445447.

11 Neufeld G, Mumblat Y, Smolkin T, Toledano S, Nir-Zvi I, et al. The role of the semaphorins in cancer. Cell Adh Migr 2016;10:652-674.

12. Vaitkienė P, Skiriutė D, Steponaitis G, Skauminas K, Tamašauskas A, et al. High level of Sema3C is associated with glioma malignancy. Diagn Pathol 2015;10:1.

13. Louis DN, Perry A, Reifenberger G, von Deimling A, Figarella-Branger D, et al. The 2016 World Health Organization Classification of Tumors of the Central Nervous System: a summary. Acta Neuropathol 2016;131:803-20.

14. Siegel R, Naishadham D, Jemal A. Cancer statistics, 2013. 
CA Cancer J Clin 2013;63:11-30.

15. Lee K-J, Joo W-I, Rha H-K, Park H-K, Chough J-K, et al. Peritumoral brain edema in meningiomas: correlations between magnetic resonance imaging, angiography, and pathology. Surg Neurol 2008;69:350-355.

16. Otsuka S, Tamiya T, Ono Y, Michiue H, Kurozumi K, et al. The relationship between peritumoral brain edema and the expression of vascular endothelial growth factor and its receptors in intracranial meningiomas. J Neurooncol 2004;70:349-357.

17. Nam DH, Lee SK, Whang SH, Shin HJ, Lee JI, et al. A study of the effects of clinicobiological factors upon the meningioma-associated peritumoral edema formation. J Korean Neurosurg Soc 1998;27:453-459.

18. Luo Y, Raible D, Raper JA. Collapsin: a protein in brain that induces the collapse and paralysis of neuronal growth cones. Cell 1993;75:217-227.

19. Acevedo LM, Barillas S, Weis SM, Göthert JR, Cheresh DA. Semaphorin 3A suppresses VEGF-mediated angiogenesis yet acts as a vascular permeability factor. Blood 2008;111:26742680.

20. Kim B-W, Kim M-S, Kim S-W, Chang C-H, Kim O-L. Peritumoral brain edema in meningiomas: correlation of radiologic and pathologic features. J Korean Neurosurg Soc 2011;49:26-30.

21. Barresi V, Vitarelli E, Cerasoli S. Semaphorin3A immunohistochemical expression in human meningiomas: correlation with the microvessel density. Virchows Arch 2009;454:563-571.

22. Gu C, Giraudo E.The role of semaphorins and their receptors in vascular development and cancer. Exp Cell Res 2013;319:1306 - 1316.

23. Rieger J, Wick W, Weller M. Human malignant glioma cells express semaphorins and their receptors, neuropilins and plexins. Glia 2003;42: $379-389$

24. Miyato H, Tsuno NH, Kitayama J. Semaphorin $3 \mathrm{C}$ is involved in the progression of gastric cancer. Cancer Sci 2012;103: $1961-1966$

25. Banu N, Teichman J, Dunlap-Brown M, Villegas G, Tufro A. Semaphorin $3 \mathrm{C}$ regulates endothelial cell function by increasing integrin activity. FASEB J 2006;20:2150-2152.

26. Yang WJ, Hu J, Uemura A, Tetzlaff F, Augustin HG, et al. Semaphorin-3C signals through Neuropilin-1 and PlexinD1 receptors to inhibit pathological angiogenesis. EMBO Mol Med 2015;7:1267-1284.

27. Oh H, Takagi H, Otani A, Koyama S, Kemmochi S, et al. Selective induction of neuropilin-1 by vascular endothelial growth factor (VEGF): a mechanism contributing to VEGFinduced angiogenesis. Proc Natl Acad Sci USA 2002; 99 : $383-388$.

28. Maione F, Molla F, Meda C, Latini R, Zentilin L, et al. Semaphorin $3 \mathrm{~A}$ is an endogenous angiogenesis inhibitor that blocks tumor growth and normalizes tumor vasculature in transgenic mouse models. J clin Invest 2009;119:3356-3372.

29. Neufeld G, Sabag AD, Rabinovicz N, Kessler O. Semaphorins in angiogenesis and tumor progression. Cold Spring Harb Perspect Med 2012;2:a006718.

30. Li K, Chen M, Li L, Lu M, Shao C, et al. The predictive value of semaphorins 3 expression in biopsies for biochemical recurrence of patients with low-and intermediate-risk prostate cancer. Neoplasma 2012;60:683-689.

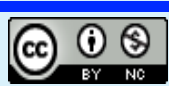

This work is licensed under a Creative Commons AttributionNon Commercial 4.0 International License. 